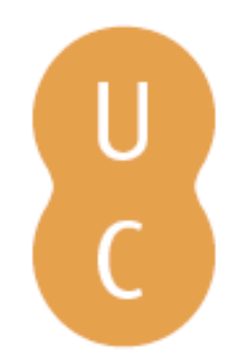

\title{
pommalina
}

\section{Systemic Clinical Outcome Routine Evaluation: SCORE15}

Autor(es): $\quad$ Vilaça, Margarida; Silva, José Tomás da; Relvas, Ana Paula

Publicado por: Imprensa da Universidade de Coimbra

URL

persistente:

URI:http://hdl.handle.net/10316.2/35898

DOI:

DOl:http://dx.doi.org/10.14195/978-989-26-0839-6_1

Accessed : $\quad$ 26-Apr-2023 09:16:27

A navegação consulta e descarregamento dos títulos inseridos nas Bibliotecas Digitais UC Digitalis, UC Pombalina e UC Impactum, pressupõem a aceitação plena e sem reservas dos Termos e Condições de Uso destas Bibliotecas Digitais, disponíveis em https://digitalis.uc.pt/pt-pt/termos.

Conforme exposto nos referidos Termos e Condições de Uso, o descarregamento de títulos de acesso restrito requer uma licença válida de autorização devendo o utilizador aceder ao(s) documento(s) a partir de um endereço de IP da instituição detentora da supramencionada licença.

Ao utilizador é apenas permitido o descarregamento para uso pessoal, pelo que o emprego do(s) título(s) descarregado(s) para outro fim, designadamente comercial, carece de autorização do respetivo autor ou editor da obra.

Na medida em que todas as obras da UC Digitalis se encontram protegidas pelo Código do Direito de Autor e Direitos Conexos e demais legislação aplicável, toda a cópia, parcial ou total, deste documento, nos casos em que é legalmente admitida, deverá conter ou fazer-se acompanhar por este aviso. 


\title{
AVALIAÇÃO FAMILIAR IN IVREVENGGio \\ FUNCIONAMENTO E VOL. I FAMILIAR
}

(2) 1.57

\begin{abstract}
Vol. 1
\end{abstract}

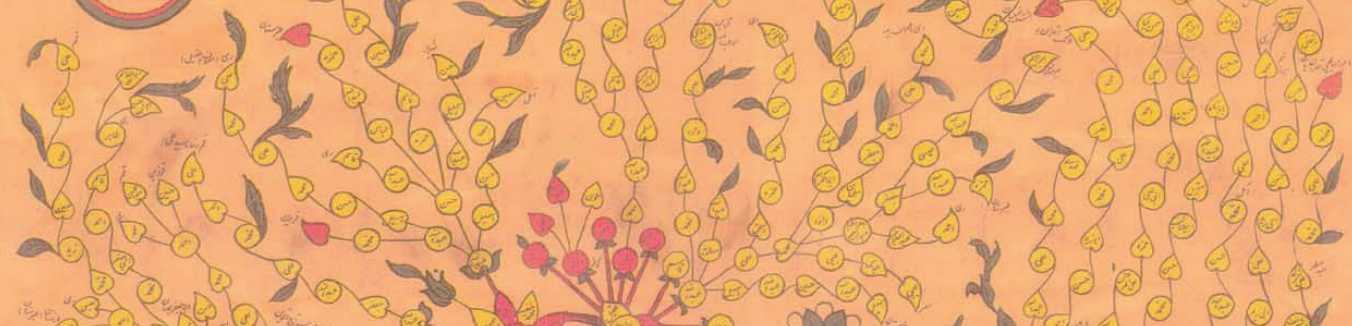
a

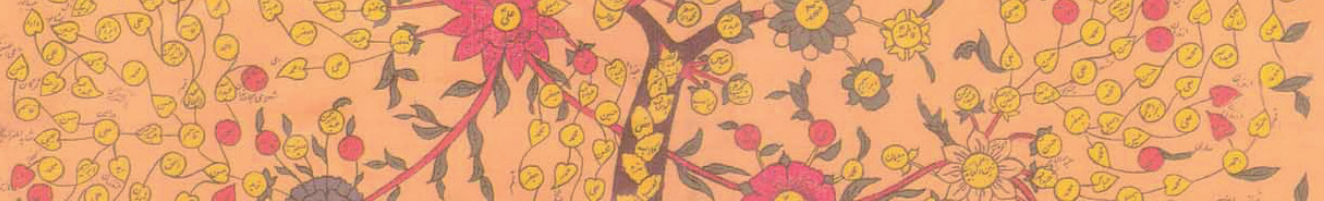

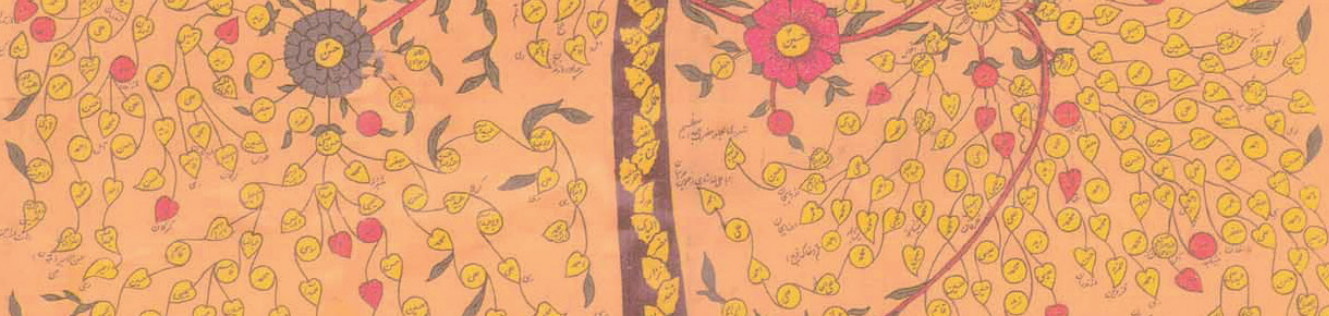

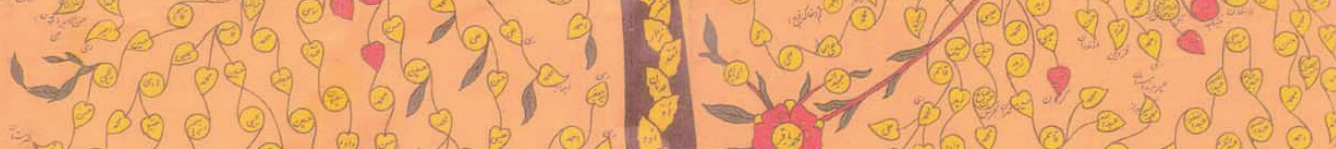
792.00

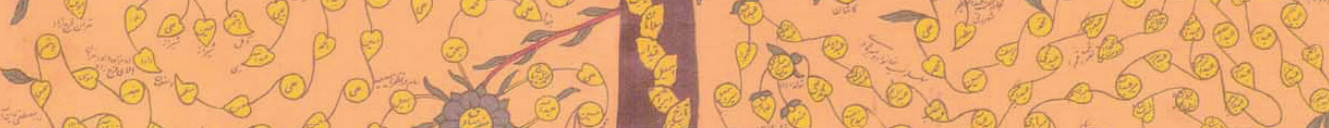

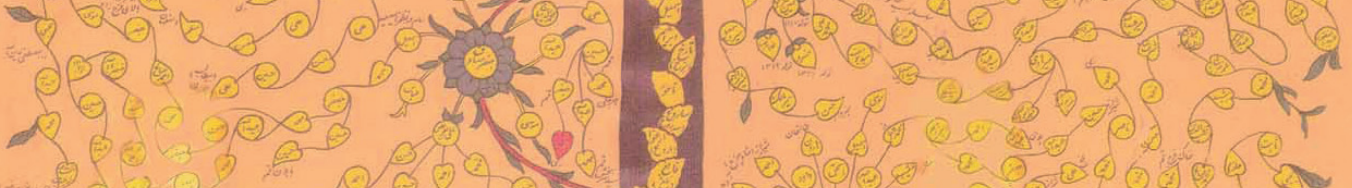

$1070-605$

ANA PAULA RELVAS UU, H(COOŔDENACÃO) 


\section{SYSTEMIC CLINICAL OUTCOME ROUTINE EVALUATION ( S C O R E - 1 5)}

Margarida Vilaça José Tomás da Silva

Ana Paula Relvas

"The SCORE approach starts from the systemic belief that the ways that relationships operate within the family are central to the welfare of all family members."

(Stratton et al., 2014, p. 4)

\section{Resumo}

Em 2010, Stratton, Bland, Janes e Lask desenvolvem um instrumento breve composto por 15 itens que permitem avaliar vários aspetos do funcionamento familiar, sensíveis à mudança terapêutica, o Systemic Clinical Outcome and Routine Evaluation (SCORE-15). Neste capítulo apresentam-se os estudos de adaptação e validação da versão portuguesa do SCORE-15 numa amostra combinada (comunitária e clínica) de 513 participantes de todo o país. A análise fatorial exploratória indica-nos uma solução fatorial de três fatores, tal como a estrutura da versão original, embora o agrupamento dos itens não replique a solução fatorial original. $\mathrm{Na}$ análise confirmatória verificou-se uma correspondência exata com os fatores da escala original: Recursos Familiares, Comunicação na Família e Dificuldades Familiares. A versão portuguesa do SCORE-15 evidencia 
igualmente uma boa consistência interna em termos da escala global e das suas dimensões. Os resultados são discutidos com base na utilidade do SCORE-15 para a investigação e prática clínica.

Palavras-chave: funcionamento familiar, análise fatorial exploratória, análise fatorial confirmatória, SCORE-15.

\begin{abstract}
In 2010, Stratton, Bland, Janes and Lask developed a brief measure composed by 15 items to evaluate several indicators of family functioning, sensitive to therapeutic change, the Systemic Clinical Outcome and Routine Evaluation (SCORE-15). In this chapter we present the adaptation and validation studies of the Portuguese version of SCORE-15 in a combined sample (community and clinical) of 513 participants from all over the country. Factor analysis revealed a three-factor solution, as the original factor structure, although the items combination does not replies the original factor solution. Confirmatory factor analysis indicated an exact correspondence with the original scale dimensions: Family Strengths, Family Communication and Family Difficulties. Portuguese SCORE-15 also showed good internal reliability for the global scale and its dimensions. Results are discussed concerning the utility of SCORE-15 both for research and clinical practice.
\end{abstract}

Key-words: family functioning;, exploratory factor analysis, confirmatory factor analysis, SCORE-15. 


\section{Instrumento}

\section{O que é, o que avalia e a quem se aplica?}

No Quadro 1 encontra-se a ficha técnica relativa ao Systemic Clinical Outcome Routine Evaluation (SCORE-15; Stratton, Bland, Janes, \& Lask, 2010).

Quadro 1.

Ficha técnica do SCORE-15

\begin{tabular}{|c|c|c|c|}
\hline O que é? & \multicolumn{3}{|c|}{$\begin{array}{l}\text { A versão portuguesa de } 15 \text { itens do SCORE - Systemic Clinical } \\
\text { Outcome Routine Evaluation (SCORE-15), escala publicada origi- } \\
\text { nalmente em } 2010 \text { por P. Stratton, J. Bland, E. Janes e J. Lask, em } \\
\text { Inglaterra }\end{array}$} \\
\hline \multirow{6}{*}{ O que avalia? } & \multicolumn{3}{|c|}{$\begin{array}{l}\text { O SCORE-15 é um questionário de auto-resposta que avalia vários } \\
\text { aspetos do funcionamento familiar que são sensíveis à mudança } \\
\text { terapêutica, contendo itens que se distribuem por três dimensões da } \\
\text { família: Recursos Familiares, Comunicação na Família e Dificuldades } \\
\text { Familiares }\end{array}$} \\
\hline & \multicolumn{3}{|l|}{ Estrutura do SCORE-15 } \\
\hline & Subescala & Número Itens & Descrição \\
\hline & $\begin{array}{l}\text { Recursos Familiares } \\
\text { (RF) }\end{array}$ & 5 & $\begin{array}{l}\text { Refere-se aos } \\
\text { recursos e à } \\
\text { capacidade } \\
\text { de adaptação } \\
\text { da família }\end{array}$ \\
\hline & $\begin{array}{l}\text { Comunicação na Família } \\
\text { (CF) }\end{array}$ & 5 & $\begin{array}{l}\text { Avalia a } \\
\text { comunicação } \\
\text { no sistema } \\
\text { familiar }\end{array}$ \\
\hline & $\begin{array}{l}\text { Dificuldades Familiares } \\
\text { (DF) }\end{array}$ & 5 & $\begin{array}{l}\text { Remete para } \\
\text { a sobrecarga } \\
\text { das dificulda- } \\
\text { des no siste- } \\
\text { ma familiar }\end{array}$ \\
\hline A quem se aplica? & \multicolumn{3}{|c|}{$\begin{array}{l}\text { O SCORE- } 15 \text { foi desenvolvido para ser utilizado com os vários } \\
\text { elementos da família com mais de } 12 \text { anos, destinando-se a uma } \\
\text { utilização corrente na prática clínica e investigação }\end{array}$} \\
\hline Como ter acesso? & \multicolumn{3}{|c|}{$\begin{array}{l}\text { O acesso ao SCORE- } 15 \text { pode ser efetuado através da página http:// } \\
\text { www.fpce.uc.pt/avaliaçaofamiliar que contém todos os instrumentos } \\
\text { de avaliação apresentados neste livro. Os utilizadores deverão facul- } \\
\text { tar os contactos pessoais e institucionais, bem como dados acerca } \\
\text { do propósito da utilização do SCORE-15 (e.g., investigação, prática } \\
\text { clínica) e concordar com as condições de utilização e de partilha } \\
\text { dos resultados com os autores da versão portuguesa }\end{array}$} \\
\hline
\end{tabular}




\section{Fundamentação e bistória}

Desde o início do século XX que terapeutas, clientes e supervisores procuram legitimar a eficácia da terapia sistémica, considerando imprescindível a evidência e auditoria desta prática profissional (Asen, 2002; Friedlander, Wildman, Heatherington, \& Skowron, 1994; Stratton, 2008). Apesar de a sua eficácia ser já amplamente reconhecida e aceite pelo público em geral, e pela comunidade científica em particular, alguns autores verificaram que a terapia familiar sistémica (TFS) se encontrava em desvantagem quando comparada com outras formas de intervenção, pelo simples facto de os profissionais não avaliarem objetivamente os seus resultados terapêuticos e as medidas de avaliação existentes serem inadequadas (Stratton et al., 2010). Até então, os instrumentos aplicados em contexto de terapia eram predominantemente direcionados para a avaliação individual e a grande maioria das escalas validadas, destinadas à avaliação familiar, não eram consistentes com a abordagem sistémica (Stratton, McGovern, Wetherell, \& Farrington, 2006), justificando assim a necessidade crescente de desenvolver uma medida capaz de avaliar o funcionamento das famílias em terapia.

Mais recentemente, têm sido propostos alguns instrumentos de avaliação dos resultados da terapia familiar, através da avaliação do funcionamento familiar, embora sejam pouco utilizados na prática clínica devido às limitações que apresentam. Exemplo disso é o Systemic Therapy Inventory of Changes (STIC; Pinsof et al., 2009) que avalia quer o funcionamento dos indivíduos (adultos e crianças), quer o de casais ou famílias, bem como o funcionamento das famílias de origem. O Synergic Navigation System (SNS; Schiepek \& Strunk, 2010), por sua vez, propõe aos clientes a realização de avaliações diárias do seu estado psicológico numa plataforma online, as quais serão posteriormente transmitidas aos terapeutas e investigadores, permitindo assim o acesso em tempo real à perspetiva dos clientes, bem como a monitorização dos processos de mudança terapêutica. Contudo, a morosidade da aplicação de ambos os instrumentos (o seu preenchimento demora cerca de uma hora para cada cliente) apontou para a necessidade de se desenvolverem instrumentos breves de avaliação dos resultados terapêuticos (Stratton et al., 2010). 
Perante este panorama, um grupo de terapeutas ingleses reuniu-se com o objetivo de desenvolver uma medida de avaliação dos resultados da terapia familiar, compatível com a linha do pensamento do construcionismo social e com a prática corrente da TFS, resultando no desenvolvimento do SCORE - Systemic Clinical Outcome and Routine Evaluation (Stratton et al., 2010). Tendo como ponto de partida uma revisão dos instrumentos de avaliação existentes até à data (Janes, 2005), bem como uma revisão dos estudos empíricos e observações de terapeutas familiares sobre o funcionamento familiar, os autores constataram que os indicadores da qualidade do funcionamento familiar refletem as melhorias mais importantes na terapia familiar. Desta forma, os autores visavam especificamente: (a) criar uma medida particularmente sensível à mudança do funcionamento familiar ao longo da terapia; (b) identificar aspetos em que os sujeitos demonstrassem mais dificuldades no seu dia-a-dia familiar; e (c) avaliar aspetos do funcionamento familiar que, expectavelmente, mudam ao longo da terapia e à medida que a família começa a lidar melhor com os problemas apresentados (Stratton et al., 2010).

Inicialmente, foi desenvolvida uma versão preliminar do SCORE com 40 itens (SCORE-40), envolvendo a colaboração de uma rede de vários clínicos e instituições de Inglaterra. Esta equipa de investigação começou por desenvolver um longo processo de revisão da literatura sobre o funcionamento familiar e a sua avaliação, de forma a identificar as dimensões mais relevantes na avaliação deste constructo. Deste processo surgiram cinco diferentes dimensões do funcionamento familiar: (a) Atmosfera e Ambiente, (b) Conflito, (c) Expressividade e Comunicação, (d) Regras e Papéis, e (e) Funcionalidade e Adaptação. De seguida, a equipa de investigação procedeu à criação dos itens, desenvolvidos com base nas sugestões dos terapeutas e participantes de estudos piloto realizados anteriormente (Stratton et al., 2010). O SCORE-40 foi então aplicado a uma amostra clínica $(N=482)$ e não-clínica $(N=126)$ para efeitos comparativos. Tanto na amostra clínica como na não-clínica, o resultado total da escala demonstrou possuir uma elevada consistência interna, traduzida num coeficiente alfa de Cronbach de .93 e .90 , respetivamente. A análise fatorial exploratória do SCORE-40 apontou para a existência de três fatores, com 
possibilidade de se considerar um quarto: (a) Competências e Adaptação, (b) Dificuldades, (c) Comunicação Disruptiva, e (d) Hostilidade e Agressão. Apesar de apresentar propriedades psicométricas adequadas, o SCORE-40 parecia ainda não ser viável para um uso corrente na prática clínica devido à sua extensão e ao tempo dispendido no seu preenchimento (Stratton et al., 2010). Atendendo a esta limitação, continuaram a realizar-se vários estudos tendo como objetivo principal o afinamento e redução dos itens do instrumento. Este processo de seleção de itens e de uma estrutura fatorial representativa de uma versão reduzida do SCORE originou o SCORE-15. A versão de 15 itens é composta por cinco itens para cada um dos três fatores: (a) Recursos Familiares, (b) Comunicação na Família e (c) Dificuldades Familiares. As análises realizadas com a amostra combinada de 608 participantes (amostra clínica e amostra não-clínica), indicaram o seguinte: o 15 itens possuem uma boa consistência interna (alfa de Cronbach de .89); a existência de três fatores; a capacidade destes 15 itens representarem o SCORE-40 original, permitindo explicar $95 \%$ da variância na média dos 40 itens (Stratton et al., 2010).

Num outro estudo realizado na Irlanda, com recurso ao SCORE-40, Cahill, O'Reilly, Carr, Dooley e Stratton (2010) confirmam novamente a estrutura de três fatores, agora com uma nova designação: (a) Competências, (b) Dificuldades e (c) Comunicação. Neste estudo, procedeu-se também ao refinamento desta solução fatorial, eliminando os itens com menores saturações nos fatores, menores comunalidades e saturações elevadas em mais que um fator, originando assim uma versão de 28 itens - o SCORE-28. À semelhança das versões anteriores, também esta revela boas qualidades psicométricas para o resultado total (e.g., coeficiente alfa de Cronbach de .93, estabilidade teste-reteste de .89, $p<.001)$. Mais recentemente, foi criada uma versão de 29 itens do SCORE, constituída por todos os itens do SCORE-28 (14 deles comuns ao SCORE-15) mais um item exclusivo do SCORE-15 (item 4). Esta versão, criada por Fay e colaboradores (2013), tem como objetivo possibilitar a recolha de informação para as duas versões, através de uma única aplicação. As análises fatoriais e confirmatórias certificam que, em ambas as versões (SCORE-15 e 28), a solução de três fatores é aquela que melhor se adequa, tendo em conta os itens e o que 
eles avaliam, indo ao encontro da solução de três fatores apoiada pelos estudos anteriores. Em todas as versões do SCORE os itens são cotados numa escala de Likert com 5 níveis de resposta $(1=$ muito bem; $2=$ bem; $3=$ em parte $4=$ mal; $5=$ muito mal $)$ ou 6 pontos $(1=$ extremamente bem; $2=$ muito bem; $3=$ bem; $4=$ em parte; $5=$ mal; $6=$ muito mal), existindo também algumas perguntas de resposta aberta direcionadas para o processo terapêutico em si, expectativas dos clientes, descrição do problema e opiniões (Fay et al., 2013).

As várias versões originais foram alvo de estudos de adaptação e validação psicométrica, indicando que a versão de 15 itens é a mais viável para uso clínico, permitindo aceder a indicadores da necessidade de terapia, bem como referentes à mudança terapêutica (Stratton et al., 2014). Atualmente, o SCORE está a ser traduzido e estudado em vários países europeus, incluindo em Portugal, onde não existem ainda escalas que se debrucem especificamente sobre a avaliação dos resultados da TFS.

\section{Estudos em Portugal}

\section{Como foi desenvolvido/adaptado e validado?}

\section{Estudos de tradução e adaptação}

Os estudos de adaptação e validação do SCORE-15 para o contexto português resultam de um processo, decorrido entre outubro de 2010 e maio de 2013, que poderá ser apresentado em duas etapas complementares: (a) tradução e adaptação cultural e (b) estudos de evidência de validade e precisão da escala.

No âmbito do projeto SCORE da European Family Therapy Association (EFTA), responsável pelo desenvolvimento e estudo psicométrico do instrumento, uma equipa de investigadores da Faculdade de Psicologia e Ciências da Educação da Universidade de Coimbra (FPCE-UC) da área de Sistémica, Saúde e Família integrou uma rede de países europeus 
que ambicionavam adaptar e validar uma medida de avaliação do funcionamento familiar para o seu país, com eventual validade europeia. Neste contexto, iniciámos o estudo de tradução do SCORE-15, tendo como base o processo de tradução-retroversão (Gjersing, Caplehorn, \& Clausen, 2010), de acordo com o protocolo de tradução proposto pelos autores do instrumento. Num primeiro momento, foram desenvolvidas duas traduções, de modo independente, por um tradutor fluente em português e inglês e por um psicólogo com formação em Psicologia clínica sistémica. De seguida, tendo como ponto de partida as traduções iniciais, quatro tradutores, com competências linguísticas semelhantes, realizaram novas traduções e promoveram a discussão dos itens, resultando numa tradução conciliadora. Por fim, dois tradutores independentes efetuaram duas retroversões, que estiveram na base da versão conciliadora final, ultimada pela equipa de investigação do SCORE (SCORE-15; Tradução portuguesa: Relvas, Vilaça, Sotero, Cunha, \& Portugal, 2010).

Realizou-se um estudo preliminar da escala, de modo a avaliar a sua adequação semântica e explorar alguns indicadores do seu funcionamento psicométrico na população portuguesa. A versão final preliminar foi então administrada a uma amostra comunitária de 21 sujeitos representantes de diferentes famílias, tendo sido pedido que registassem eventuais erros, desadequações, ambiguidades na formulação dos itens ou outras dificuldades sentidas ao responder ao SCORE-15. Deste procedimento resultaram pequenas alterações na versão administrada, nomeadamente, a alteração da formulação de itens potencialmente ambíguos (itens 5 , 12 e 13) e a clarificação das instruções de preenchimento (Portugal, Sotero, Cunha, Vilaça, \& Relvas, 2010). De um modo geral, este estudo piloto indicou-nos que o SCORE-15 é um questionário acessível aos respondentes e de rápida aplicação (o seu preenchimento demorou entre 5 a 10 minutos).

Concluído o estudo de tradução e adaptação para o contexto português, procedemos à administração do SCORE-15 a um conjunto alargado de famílias de dois contextos distintos: contexto clínico (clientes de TFS) e não-clínico (amostra comunitária). À semelhança dos estudos de validação 
do SCORE-28 para a população irlandesa (Cahill et al., 2010), a amostra utilizada neste estudo consiste numa amostra combinada $(N=513)$, ou seja, trata-se de uma amostra que integra uma subamostra comunitária e uma subamostra clínica, recolhida por todo o país (continente e ilhas), entre os meses de novembro de 2010 e fevereiro de 2013.

A subamostra comunitária $(n=406)$ foi recolhida através de dois procedimentos distintos: versão em papel e lápis e versão online, tendo em consideração os seguintes critérios de inclusão: (a) idade dos sujeitos superior a 12 anos, (b) serem de nacionalidade portuguesa, (c) não estarem a receber apoio psiquiátrico, e (d) não serem amigos próximos ou familiares dos investigadores. Dadas as dificuldades inicialmente sentidas na recolha de protocolos através da versão do SCORE em formato papel e lápis, optou-se por desenvolver uma plataforma online. Mais especificamente, foram recolhidos 244 protocolos através da modalidade "papel-lápis" (60.1\%) e 162 através da modalidade online (39.9\%). A subamostra clínica $(n=107)$ contou com a participação de diversos centros de terapia (públicos e privados) que realizam terapia familiar e/ou de casal, distribuídos pelo país, especificamente nas regiões Norte, Centro, Lisboa, Região Autónoma da Madeira e Região Autónoma dos Açores. À subamostra clínica correspondem protocolos aplicados às famílias no início da primeira sessão de terapia.

Em ambos os casos, para além da aplicação do SCORE-15, utilizou-se também um questionário de dados sociodemográficos e familiares, para uma identificação e caracterização do respondente e da sua família. Este questionário averigua alguns dados pessoais do respondente, como o sexo, a idade, o estado civil, a profissão, a nacionalidade, entre outras variáveis sociodemográficas, mas também permite recolher dados de natureza familiar, como informações sobre os elementos do agregado familiar. Antes de se proceder à aplicação dos instrumentos, foi apresentado aos sujeitos que aceitaram participar no estudo um documento de consentimento informado que continha a apresentação dos objetivos do estudo, a garantia do anonimato das respostas, o caráter voluntário da participação e assegurava que os dados seriam utilizados somente para fins estatísticos. 
A amostra combinada do nosso estudo é então composta por 297 participantes do sexo feminino (57.9\%) e 216 do sexo masculino (42.1\%). Quanto às idades, estas encontram-se compreendidas entre os 12 e os 92 anos, sendo a média $38.57(D P=16.32)$. As faixas etárias mais predominantes são 12-24 (21.6\%) e 40-49 anos (20.9\%). A escolaridade dos participantes é diversificada, variando entre os indivíduos sem escolaridade (2.1\%) e os doutorados (1.2\%), sendo a maioria licenciados (34.3\%). Relativamente ao estado civil, os sujeitos, na sua maioria, são casados ou vivem em união de facto (49.7\%), seguindo-se os solteiros (38.4\%) e, menos frequente, os divorciados, separados e viúvos (11.9\%). Em termos do nível socioeconómico (NSE) da amostra, aferido com base na classificação de Simões (2000), que cruza dados relativos às profissões e à escolaridade, a categoria mais representativa é o NSE médio (34.9\%), seguindo-se o NSE elevado (22.0\%) e, por último o NSE baixo (1.8\%) (Simões, 2000). No que à zona de residência diz respeito, os 513 sujeitos respondentes, agrupam-se, na sua grande maioria, em áreas predominantemente urbanas (48.1\%), seguindo-se os habitantes de áreas moderadamente urbanas (30.6\%) e predominantemente rurais $(8.0 \%)$ (INE, 2002). Por último, em termos da

posição e composição do agregado familiar dos sujeitos participantes, a maioria são filhos ou filhas (33.4\%) e mães (28.3\%) pertencentes a agregados familiares compostos, em média, por três a quatro elementos.

Após a conclusão da tradução portuguesa do SCORE-15 e da realização de estudos de adaptação desta escala, têm sido desenvolvidos estudos de investigação com este instrumento na FPCE-UC, especificamente, sobre o processo e resultados em TFS.

\section{Estudos descritivos}

A análise descritiva dos 15 itens do SCORE encontra-se no Quadro 2, onde estão apresentados os valores para a média, desvio-padrão, moda, amplitude, assimetria e curtose. São também apresentadas as análises de consistência interna, nomeadamente, os valores das correlações item-total e os valores do coeficiente alfa de Cronbach quando o item é excluído. 
Quadro 2.

Estatísticas descritivas dos itens do SCORE-15 e consistência interna

\begin{tabular}{lcccccccc}
\hline Item & M & DP & Moda & Amplitude & Assimetria & Curtose & $\begin{array}{c}\text { Correlação } \\
\text { Item-Total } \\
\text { Corrigida }\end{array}$ & $\begin{array}{c}\text { Alfa com } \\
\text { Item Ex- } \\
\text { cluído }\end{array}$ \\
\hline 1 & 1.89 & 0.94 & 1 & $1-5$ & 0.99 & 0.69 & .09 & .85 \\
2 & 3.05 & 1.31 & 3 & $1-5$ & -0.02 & -1.09 & .62 & .83 \\
3 & 1.97 & 0.99 & 1 & $1-5$ & 0.85 & 0.18 & .15 & .85 \\
4 & 3.14 & 1.46 & 5 & $1-5$ & -0.10 & -1.33 & .68 & .82 \\
5 & 3.12 & 1.16 & 3 & $1-5$ & -0.11 & -0.75 & .17 & .85 \\
6 & 1.75 & 0.91 & 1 & $1-5$ & 1.20 & 1.11 & .12 & .85 \\
7 & 3.11 & 1.59 & 5 & $1-5$ & -0.09 & -1.57 & .70 & .82 \\
8 & 3.07 & 1.34 & 3 & $1-5$ & -0.05 & -1.14 & .61 & .83 \\
9 & 3.17 & 1.33 & 3 & $1-5$ & -0.07 & -1.18 & .69 & .82 \\
10 & 1.93 & 0.96 & 1 & $1-5$ & 0.92 & 0.43 & .16 & .85 \\
11 & 3.05 & 1.35 & 3 & $1-5$ & -0.03 & -1.18 & .74 & .82 \\
12 & 3.08 & 1.53 & 5 & $1-5$ & -0.04 & -1.49 & .73 & .82 \\
13 & 3.13 & 1.08 & 3 & $1-5$ & -0.06 & -0.62 & .34 & .84 \\
\hline 15 & 3.02 & 1.41 & $2^{\mathrm{a}}$ & $1-5$ & 0.01 & -1.29 & .73 & .82 \\
\hline 0 & 2.32 & 0.94 & 2 & $1-5$ & 0.43 & 0.04 & .16 & .85 \\
\hline
\end{tabular}

Nota. Os valores assinalados a itálico correspondem a itens com valores inferiores ao desejável (.30).

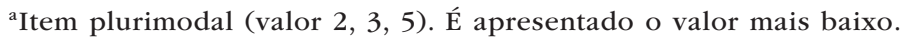

Os resultados encontrados (cf. Quadro 2) indicam que o item que apresenta uma média mais elevada $(M=3.17 ; D P=1.33)$ corresponde ao item 9 "Na minha família, parece que surgem crises umas atrás das outras". Por outro lado, o item 6 "Confiamos uns nos outros" obteve a média mais baixa $(M=1.75 ; D P=0.91)$. De um modo geral, os valores oscilam entre 1 e 5 , sendo que o valor mais frequente é o 3 ("Descreve-nos em Parte"). Verifica-se ainda que os inquiridos utilizaram, para cada um dos 15 itens, as cinco possibilidades de resposta existentes. Quanto à assimetria, os itens apresentam na sua maioria um valor negativo, com destaque para um maior afastamento do item 6 (assimetria $=1.20$ ). Em termos do grau de achatamento da distribuição, os itens que se encontram mais afastados do valor zero são os itens $2,4,6,7,8,9,11,12$ e 14, com um valor positivo para o item 6 (curtose $=1.11$ ) e valores negativos para os restantes itens, entre -1.09 e -1.57 . 


\section{Estudos de precisão}

Para averiguar a precisão dos dados, procedemos à análise da consistência interna dos itens do SCORE-15, através do cálculo do coeficiente alfa de Cronbach, da escala total e dos fatores que a compõem. Assim sendo, a escala total apresenta uma boa consistência interna ( $\alpha=.84)$, tal como acontece com o Fator $1(\alpha=.85)$, o Fator $2(\alpha=.83)$ e o Fator 3 ( $\alpha=$.82) (Pestana \& Gageiro, 2008). A análise dos valores do coeficiente alfa da escala total aquando a exclusão de qualquer um dos itens indica-nos que a sua exclusão não aumenta de forma expressiva a consistência interna total da escala (cf. Quadro 2). Os valores de correlação item-total revelam uma adequada capacidade discriminante de todos os itens $(r>.30)$ (Wilmut, 1975), à exeção dos itens 1, 3, 5, 6, 10 e 15.

\section{Estudos de validade de constructo: Análise fatorial exploratória}

A validade interna do SCORE-15 foi verificada através da análise fatorial exploratória (análise das componentes principais das correlações entre as variáveis), uma das técnicas mais reconhecidas na identificação de constructos subjacentes. Procedemos, então, à verificação dos critérios de adequação da amostra não se tendo verificado a normalidade da distribuição dos dados $(K-S=.09 ; p<.01)$. Contudo, a dimensão da amostra é superior a 100 sujeitos $(N=513)$ e apresenta um rácio de cerca de 34 sujeitos para cada item da escala, satisfazendo assim as propostas mais exigentes relativas à dimensão amostral (Hair, Anderson, Tatham, \& Black, 1995). Obtiveram-se resultados significativos ao nível da medida de adequação da amostra para a realização da análise em componentes principais, através do cálculo de critério de Kaiser-Meyer-Olkin (KMO) (.907) e do teste de esfericidade de Bartlett $\left(\chi^{2}=4006.318 ; g l=105, p<.001\right)$, mostrando que existem correlações entre as variáveis (Pestana \& Gageiro, 2008).

A análise em componentes principais aponta para uma solução de três fatores (com valores próprios superiores a 1) que, em conjunto, explicam 64.98\% da variância total. Avançamos com a rotação Varimax, forçando a 
extração de três fatores, uma vez que este método maximiza as saturações mais elevadas e reduz as saturações mais baixas (Pestana \& Gageiro, 2008), permitindo assim comparar os resultados com a estrutura fatorial sugerida pelos autores da escala. Da observação do Quadro 3, constatamos que, apesar de os resultados indicarem uma estrutura fatorial semelhante à original, i.e., dividida em três fatores, os itens não se encontram agrupados nos mesmos fatores, não se confirmando uma correspondência com a escala original, em termos estatísticos e teóricos. Analisando o conteúdo dos itens de cada um dos três fatores, nota-se que, à exceção do segundo fator (F2) (onde estão agrupados todos os itens relativos à dimensão Recursos Familiares), os restantes dois fatores reúnem itens que claramente não se enquadram na definição desses componentes. Especificamente, num primeiro fator (F1) saturam oito itens, sendo quatro relativos à subescala Comunicação na Família (itens 2, 4, 8 e 12) e outros quatro respeitantes às Dificuldades Familiares (itens 7, 9, 11 e 14). No terceiro fator (F3) encontramos apenas dois itens, sendo um item relativo à Comunicação na Família (saturando simultaneamente no primeiro fator) e um outro referente às Dificuldades Familiares (cf. Quadro 3).

Quadro 3.

Matriz rodada, comunalidades e variância explicada (Rotação Varimax) - SCORE-15

\begin{tabular}{|c|c|c|c|c|}
\hline \multirow[t]{2}{*}{ Itens } & \multicolumn{3}{|c|}{ Fatores } & \multirow{2}{*}{$b^{2}$} \\
\hline & 1 & 2 & 3 & \\
\hline 14. Na minha família culpamo-nos... & .866 & & & .751 \\
\hline 12. As pessoas da minha família... & .860 & & & .741 \\
\hline 11. As coisas parecem correr... & .845 & & & .734 \\
\hline 7. Sentimo-nos muito infelizes... & .820 & & & .675 \\
\hline 4. Sinto que é arriscado discordar... & .820 & & & .682 \\
\hline 9. Na minha família parece que... & .771 & & & .644 \\
\hline 8. Na minha família, quando as pessoas... & .766 & & & .614 \\
\hline 2. Na minha família muitas vezes... & .747 & & & .593 \\
\hline 10. Quando um de nós está... & & .838 & & .708 \\
\hline 3. Todos nós somos ouvidos... & & .801 & & .693 \\
\hline 1. Na minha família, falamos... & & .785 & & .627 \\
\hline 15. Somos bons a encontrar... & & .759 & & .579 \\
\hline 6. Confiamos uns... & & .747 & & .568 \\
\hline 5. Sentimos que é difícil... & & & .906 & .829 \\
\hline 13. Na minha família as pessoas... & .358 & & .426 & .310 \\
\hline \% variância explicada & 36.251 & 20.863 & 6.869 & \\
\hline
\end{tabular}


A solução fatorial resultante revela-se, desta forma, complexa e ambígua: para além de não fazer sentido teoricamente, a distribuição alcançada não coincide com os três fatores sugeridos pelos autores da escala, não indo, por isso, ao encontro dos resultados alcançados com os outros estudos de validação realizados com o SCORE-15 (Cahil et al., 2010; Fay et al., 2013).

Estudos de validade de constructo: Análise fatorial confirmatória

Foram realizados estudos de análise fatorial confirmatória do modelo proposto pelos autores da versão original do SCORE-15 de modo a averiguar, mais uma vez, a correspondência dos nossos dados. Ou seja, testámos um modelo composto por três fatores (Recursos Familiares, Comunicação na Família e Dificuldades Familiares) constituídos cada um por cinco itens (RF: itens 1, 3, 6, 10 e 15; CF: itens 2, 4, 8, 12 e 13; DF: itens 5, 7, 9, 11 e 14).

A análise dos resultados indica que a estrutura trifatorial original apresenta índices de ajustamento adequados para o modelo re-especificado (cf. Quadro 4). O valor dos índices considerados para determinar a adequação do ajustamento global do modelo de medida do SCORE-15 inspecionado [qui-quadrado absoluto $(\chi 2)=215.082(p<.001)$, qui-quadrado normalizado $(\chi 2 / g l)=2.501]$ foi superior $[C F I=.967, G F I=.947]$ ao valor critério de .90. Relativamente à raiz quadrada da média do erro de aproximação $($ RMSEA $)$, o valor foi de $.057($ Lo $=.05, \mathrm{Hi}=.06)$. Uma vez que $\times 2 / g l$ é inferior a 5, o índice de $C F I$ é superior a .95 , o índice de $G F I$ é superior a .90 e RMSEA é inferior a .06 (Byrne, 2001; Maroco, 2010), concluímos que os dados se ajustam à estrutura fatorial original do SCORE-15.

Quadro 4 .

Índices de adequação dos modelos testados

\begin{tabular}{lcccc}
\hline \multicolumn{5}{c}{ Índice } \\
\hline Modelo & $\chi 2$ & GFI & CFI & RMSEA \\
\hline Inicial & $986.907(g l=90)$ & .857 & .773 & .140 \\
Re-especificado & $215.082(g l=86)$ & .947 & .967 & .054
\end{tabular}

GFI - Goodness-of-Fit Index; CFI - Comparative Fit Index; RMSEA - Root Mean Square Error of Approximation 
Para se obter este ajustamento final foram necessárias algumas alterações sugeridas pelos índices de modificação. A este respeito, note-se que apenas se realizaram alterações quando o índice de modificação era elevado e simultaneamente correspondia a uma alteração teoricamente plausível. A título de exemplo, salientamos a associação entre os fatores Comunicação e Dificuldades Familiares, pois ambos abordam obstáculos ou aspetos menos positivos da vida familiar. Um outro ajustamento realizado consiste na associação entre o item 9 ("Na minha família, parece que surgem crises umas atrás das outras") e o item 11 ("As coisas parecem correr sempre mal para a minha família”) que remetem para a vivência de obstáculos na família, percebidos sob um ponto de vista do controlo externo. O modelo final atinge uma boa adequação, demonstrando que os ajustamentos efetuados levaram a melhorias relevantes nos índices de ajustamento.

\section{Aplicação}

\section{Como aplicar, cotar e interpretar?}

O SCORE-15 poderá ser aplicado a indivíduos, casais e famílias, desde que os seus elementos tenham idade igual ou superior a 12 anos. A sua administração é recomendável em contexto clínico, uma vez que providencia breves descrições de variados aspetos do relacionamento familiar que são relevantes para indivíduos, casais ou famílias que recorrem à terapia. O instrumento deverá ser preenchido individualmente durante, antes ou após as sessões, conforme o propósito da sua aplicação. Especificamente, para efeitos de investigação no âmbito da terapia familiar sistémica breve, é sugerida a sua aplicação antes, durante ou após a realização de sessões importantes ou momentos-chave de mudança na terapia. Neste sentido, os autores sugerem a sua aplicação antes da primeira sessão, depois da quarta sessão (cerca de seis meses depois) e na sétima sessão (ou última sessão), por permitir a avaliação dos momentos correspondentes ao início, meio e final da terapia (Stratton et al., 2014). Para além disso, este instrumento poderá ainda ser utilizado com a população em 
geral, i. e., num contexto não-clínico, enquanto medida de avaliação do funcionamento familiar.

No momento do preenchimento, deverá ser respeitada a privacidade do respondente de modo a garantir a confidencialidade das informações, relativamente aos técnicos e aos familiares. Poderá ainda ser facultada ajuda aos respondentes que manifestem dificuldades ao nível da leitura, tendo o cuidado de não alterar a formulação original dos itens.

A cotação do SCORE-15 (total da escala e dimensões) poderá ser feita através de dois métodos distintos: recorrendo à inversão dos itens negativos ou analisando os itens negativos sem proceder à sua inversão. A primeira possibilidade consiste em calcular o resultado total do SCORE através da inversão dos itens $2,4,5,7,8,9,11,12,13$ e 14 para que as pontuações mais elevadas correspondam a maiores dificuldades na família. O cálculo das três dimensões que o compõem, para uma análise de informação mais específica, realiza-se somando os cinco itens correspondentes a cada dimensão e dividindo esse valor por cinco. Por exemplo, para a dimensão Comunicação na Família (CF) são somados os cinco itens invertidos e divididos por cinco $[\mathrm{CF}=(\mathrm{R} 2+\mathrm{R} 4+\mathrm{R} 8+$ $\mathrm{R} 12+\mathrm{R} 13) /$ 5].

Uma outra forma de codificar os dados do SCORE-15 total é somando todos os itens negativos $(2+4+5+7+8+9+11+12+13+14)$, subtrair o resultado deste somatório a 60 e acrescentar o total dos itens positivos $(1+3+6+10+15)-$ desta forma $[60-(2+4+5+7+8+$ $9+11+12+13+14)]+(1+3+6+10+15)]$, obtemos o resultado total para cada sujeito. Para calcularmos a média do resultado total do SCORE, dividimos por 15 . O cálculo das dimensões realiza-se através da soma dos itens, sendo que quando os itens são negativos, subtraímos o resultado a 30. Por exemplo, para o cálculo da dimensão Dificuldades Familiares retiramos a 30 o somatório dos cinco itens negativos [DF $=30-(\mathrm{R} 5+\mathrm{R} 7+\mathrm{R} 9+\mathrm{R} 11+\mathrm{R} 14)]$. Para calcularmos os resultados médios obtidos em cada uma das subescalas seguimos a mesma lógica utilizada para o total da escala, isto é, dividimos o total obtido em cada dimensão por cinco, sendo que resultados mais baixos correspondem a um melhor funcionamento. 


\section{Vantagens, limitações e estudos futuros}

O SCORE-15 revela ser um instrumento com propriedades psicométricas bastante razoáveis e apresenta uma estrutura fatorial semelhante à sua versão original. Sendo um indicador válido do funcionamento e mudança familiar, permite-nos aceder a informações relevantes sobre indivíduos, casais e famílias, que recorrem à terapia familiar, em geral, de forma prática e rápida (entre 5 a 10 minutos). Para além disso, o seu preenchimento poderá incitar os respondentes à discussão, reflexão ou partilha de informações potencialmente úteis para a terapia. Ao nível da investigação, fica disponível para a população portuguesa uma ferramenta de estudo dos resultados e processo da terapia familiar sistémica, na medida que permite monitorizar e descrever indicadores comprovados do progresso da terapia sistémica. Desenvolvido de modo a permitir que os respondentes relatem aspetos relativos à sua interação familiar, independentemente do seu meio cultural e socioeconómico, o SCORE-15 representa ainda um importante instrumento de investigação no contexto comunitário, particularmente sempre que se pretenda intervir ao nível das relações na família.

A amostra utilizada no presente estudo poderá constituir-se como uma limitação, na medida em que não é uma amostra estratificada e representativa da nossa população. Parece-nos ainda que os estudos de adaptação e validação beneficiariam de análises independentes com população clínica

e população comunitária, de modo a avaliar a capacidade discriminante do questionário e desenvolver valores normativos para ambos os contextos. Por outro lado, verificada a sua validade e aplicabilidade no contexto português, seria importante prosseguir com estudos de validade das pontuações na escala enquanto indicador da mudança terapêutica, à semelhança do que foi realizado pelos autores da versão original. No fundo, tais análises permitir-nos-iam concluir se o SCORE-15 apresenta mudanças significativas ao longo da TFS, bem como perceber de que modo se relaciona com outros indicadores do progresso terapêutico. Seria igualmente útil analisar o funcionamento da escala em diferentes momentos da terapia (sessões iniciais, intermédias, finais e de seguimento). Por fim, parece-nos importante estender a aplicabilidade da escala aos elementos mais novos 
da família, nomeadamente através da adaptação portuguesa da recente versão do SCORE-15 para crianças com idades compreendidas entre os 8 e os 11 anos (Jewell, Carr, Stratton, Lask, \& Eisler, 2013).

\section{Bibliografia}

Asen, E. (2002). Outcome research in family therapy. Advances in Psychiatric Treatment, 8, 230-238. doi:10.1192/apt.8.3.230

Byrne, B. M. (2001). Structural equation modeling with AMOS, EQS, and LISREL: Comparative approaches to testing for the factorial validity of a measuring instrument. International Journal of Testing, 1, 55-86. Acedido em http://dx.doi.org/10.1207/S15327574IJT0101_4

Cahill, P., O'Reilly, K., Carr, A., Dooley, B., \& Stratton, P. (2010). Validation of a 28-item version of the Systemic Clinical Outcome and Routine Evaluation in an Irish context: The SCORE-28. Journal of Family Therapy, 32, 210-231. doi:10.1111/j.1467-6427.2010.00506.x

Fay, D., Carr, A., O'Reilly, K., Cahill, P., Dooley, B. Guerin, F., \& Stratton, P. (2013). Irish norms for the SCORE-15 and 28 from a national telephone survey. Journal of Family Therapy, 35, 24-42. doi:10.1111/j.1467-6427.2011.00575.x

Friedlander, M., Wildman, J., Heatherington, L., \& Skowron, E. A. (1994). What we do and don't know about the process of family therapy. Journal of Family Psychology, 8, 390-416. doi:10.1037/0893-3200.8.4.390

Gjersing, L., Caplehorn, J., \& Clausen, T. (2010). Cross-cultural adaptation of research instruments: Language, setting, time and statistical considerations. BMC Medical Research Methodology, 10(13), 101-110. doi:10.1186/1471-2288-10-13

Hair, J. F. Jr., Anderson, R. E., Tatham, R. L., \& Black, W. C. (1995). Multivariate data analysis with readings $\left(4^{\text {th }}\right.$ ed.). Englewood Cliffs, NJ: Prentice Hall.

Instituto Nacional de Estatística (2002). Censos 2001: Resultados definitivos. Disponível em: http://www.ine.pt/xportal/xmain?xpid=INE\&xpgid=ine_destaques\&DESTAQUESdest_ boui $=71467 \& D E S T A Q U E S m o d o=2$

Janes, E. (2005) Self-Report Measures of Family Function and Change Following Family Therapy: A Review of Conceptual Issues, Existing Measures and Proposals for Improvement. Disponível em: www.psyc.leeds.ac.uk/staff/p.m.stratton/

Jewell, T., Carr, A., Stratton, P., Lask, J., \& Eisler, I. (2013). Development of a children's version of the SCORE index of family function and change. Family Process, 52(4), 673-684. doi: 10.1111/famp.12044

Maroco, J. (2010). Análise de equações estruturais. Lisboa: Escolar.

Pestana, M. H., \& Gageiro, J. (2008). Análise de dados para ciências sociais - A complementaridade do SPSS ( $5^{\mathrm{a}}$ ed.). Lisboa: Sílabo.

Pinsof, W. M., Zinbarg, R., Lebow, J., Knobloch-Fedders, L. M., Durbin, K. E., Chambers, A. L., ... Friedman, G. (2009). Laying the foundation for progress research in family, couple and individual therapy: The development and psychometric features of the initial systemic therapy inventory of change. Psychotherapy Research, 19(2), 143-156. doi:10.1080/10503300802669973

Portugal, A., Sotero, L., Cunha, D., Vilaça, M., \& Relvas, A. P. (2010, Outubro). SCORE-15: Exploratory study of preliminary data in a sample of Portuguese families. Comunicação apresentada no $7^{\circ}$ European Family Therapy Association Congress, Paris, França. 
Schiepek, G., \& Strunk, G. (2010). The identification of critical fluctuations and phase transitions in short term and coarse-grained time series - a method for the real-time monitoring of human change processes. Biological Cybernetics, 102, 197-207. doi:10.1007/ s00422-009-0362-1

Simões, M. (2000). Investigação no âmbito da aferição nacional do teste das Matrizes Progressivas Coloridas de Raven (M.P.C.R.). Lisboa: Fundação Calouste Gulbenkian/ Fundação para a Ciência e Tecnologia.

Stratton, P. (2008). PRN in Action: Constructing an outcome measure for therapy with relational systems: Practitioner research networks in action. The Psychotherapist, 38, 15-16.

Stratton, P., Bland, J., Janes, E., \& Lask, J. (2010). Developing an indicator of family function and a practicable outcome measure for systemic therapy and couple therapy: The SCORE. Journal of Family Therapy, 32, 232-258. doi:10.1111/j.1467-6427.2010.00507.x

Stratton, P., Lask, J., Bland, J., Nowotny, E., Evans, C., Singh, R., Janes, E., \& Peppiatt, A. (2014). Detecting therapeutic improvement early in therapy: Validation of the SCORE-15 Index of Family Functioning and Change. Journal of Family Therapy, 36(1), 3-19. doi:10.1111/1467-6427.12022

Stratton, P., McGovern, M., Wetherell, A., \& Farrington, C. (2006). Family therapy practitioners researching the reactions of practitioners to an outcome measure. Australian and New Zealand Journal of Family Therapy, 27, 199-207.

Wilmut, J. (1975). Objective test analysis: Some criteria for item selection. Research in Education, 13, 27-56. 